\title{
Aqueous Biphasic System Containing Long Chain Anion- functionalized Ionic Liquids for High-performance Extraction
}

Yuanbang Xie, Huabin Xing*, Qiwei Yang, Zongbi Bao, Baogen Su and Qilong Ren*

Key Laboratory of Biomass Chemical Engineering of Ministry of Education, College of

Chemical and Biological Engineering, Zhejiang University, Zheda Road 38, Xihu District,

Hangzhou 310027, China

Supporting Information 


\section{Part A: NMR data of the synthesized ILs}

\section{Cholinium butyrate $[\mathrm{Ch}]\left[\mathrm{C}_{3} \mathrm{H}_{7} \mathrm{COO}\right]$}

${ }^{1} \mathrm{H}$ NMR (400 MHz, $\mathrm{D}_{2} \mathrm{O}, \delta / \mathrm{ppm}$ relative to TMS): $\delta 3.91$ (ddd, $\left.J=7.8,5.3,2.9 \mathrm{~Hz}, 2 \mathrm{H}\right), 3.39$ $3.34(\mathrm{~m}, 2 \mathrm{H}), 3.05(\mathrm{~s}, 9 \mathrm{H}), 2.00(\mathrm{t}, J=7.3 \mathrm{~Hz}, 2 \mathrm{H}), 1.41(\mathrm{~h}, J=7.4 \mathrm{~Hz}, 2 \mathrm{H}), 0.74(\mathrm{t}, J=7.4 \mathrm{~Hz}$ $3 \mathrm{H})$.

${ }^{13} \mathrm{C}$ NMR (400 MHz, $\mathrm{D}_{2} \mathrm{O}, \delta / \mathrm{ppm}$ relative to TMS): $\delta 183.90(\mathrm{~s}), 67.48-67.18(\mathrm{~m}), 55.51(\mathrm{~s})$, 53.94 - $53.62(\mathrm{~m}), 39.56(\mathrm{~s}), 19.31(\mathrm{~s}), 13.20(\mathrm{~s})$.

\section{Cholinium hexanoate $[\mathrm{Ch}]\left[\mathrm{C}_{5} \mathrm{H}_{11} \mathrm{COO}\right]$}

${ }^{1} \mathrm{H}$ NMR (400 MHz, $\mathrm{D}_{2} \mathrm{O}, \delta / \mathrm{ppm}$ relative to TMS): $\delta 3.92(\mathrm{ddd}, J=7.8,5.3,2.8 \mathrm{~Hz}, 2 \mathrm{H}), 3.40$ $3.33(\mathrm{~m}, 2 \mathrm{H}), 3.06(\mathrm{~s}, 9 \mathrm{H}), 2.02(\mathrm{t}, J=7.5 \mathrm{~Hz}, 2 \mathrm{H}), 1.46-1.35(\mathrm{~m}, 2 \mathrm{H}), 1.22-1.08(\mathrm{~m}, 4 \mathrm{H})$, $0.73(\mathrm{t}, J=7.0 \mathrm{~Hz}, 3 \mathrm{H})$.

${ }^{13} \mathrm{C}$ NMR (400 MHz, $\mathrm{D}_{2} \mathrm{O}, \delta / \mathrm{ppm}$ relative to TMS): $\delta 184.13(\mathrm{~s}), 67.49-67.19(\mathrm{~m}), 55.52(\mathrm{~s})$, 53.95 - $53.63(\mathrm{~m}), 37.55(\mathrm{~s}), 30.97(\mathrm{~s}), 25.54(\mathrm{~s}), 21.75(\mathrm{~s}), 13.25(\mathrm{~s})$.

\section{Cholinium octanoate $[\mathrm{Ch}]\left[\mathrm{C}_{7} \mathrm{H}_{15} \mathrm{COO}\right]$}

${ }^{1} \mathrm{H}$ NMR (400 MHz, $\mathrm{D}_{2} \mathrm{O}, \delta / \mathrm{ppm}$ relative to TMS): $\delta 3.92$ (ddd, $\left.J=7.8,5.3,2.8 \mathrm{~Hz}, 2 \mathrm{H}\right), 3.41-$ $3.35(\mathrm{~m}, 2 \mathrm{H}), 3.06(\mathrm{~s}, 9 \mathrm{H}), 2.03(\mathrm{t}, J=7.5 \mathrm{~Hz}, 2 \mathrm{H}), 1.41$ (p, $J=7.3 \mathrm{~Hz}, 2 \mathrm{H}), 1.21-1.09(\mathrm{~m}, 8 \mathrm{H})$, $0.73(\mathrm{t}, J=6.9 \mathrm{~Hz}, 3 \mathrm{H})$.

${ }^{13} \mathrm{C}$ NMR (400 MHz, $\mathrm{D}_{2} \mathrm{O}, \delta / \mathrm{ppm}$ relative to TMS): $\delta 184.07(\mathrm{~s}), 67.50-67.19(\mathrm{~m}), 55.52(\mathrm{~s})$, 53.96 - 53.64 (m), 37.62 (s), 31.03 (s), 28.70 (s), 28.24 (s), 25.89 (s), 21.98 (s), 13.39 (s).

\section{Cholinium laurate $[\mathrm{Ch}]\left[\mathrm{C}_{11} \mathrm{H}_{23} \mathrm{COO}\right]$}

${ }^{1} \mathrm{H}$ NMR (400 MHz, $\mathrm{D}_{2} \mathrm{O}, \delta / \mathrm{ppm}$ relative to TMS): $\delta 3.92$ (ddd, $\left.J=7.8,5.3,2.8 \mathrm{~Hz}, 2 \mathrm{H}\right), 3.41$ $3.36(\mathrm{~m}, 2 \mathrm{H}), 3.08(\mathrm{~s}, 9 \mathrm{H}), 2.02(\mathrm{t}, J=7.5 \mathrm{~Hz}, 2 \mathrm{H}), 1.47-1.35(\mathrm{~m}, 2 \mathrm{H}), 1.17(\mathrm{~s}, 16 \mathrm{H}), 0.76(\mathrm{t}, J$ $=6.7 \mathrm{~Hz}, 3 \mathrm{H})$. 
${ }^{13} \mathrm{C}$ NMR (400 MHz, $\mathrm{D}_{2} \mathrm{O}, \delta / \mathrm{ppm}$ relative to TMS): $\delta 182.86(\mathrm{~s}), 67.60$ - $67.18(\mathrm{~m}), 55.55$ (s), 54.12 - $53.68(\mathrm{~m}), 37.92(\mathrm{~s}), 31.88(\mathrm{~s}), 29.87-29.24(\mathrm{~m}), 26.35(\mathrm{~s}), 22.55(\mathrm{~s}), 13.78(\mathrm{~s})$. 
Part B: Phase Diagrams and Fitting Parameters

Table S1 Phase diagram data of $[\mathrm{Ch}]\left[\mathrm{C}_{3} \mathrm{H}_{7} \mathrm{COO}\right]$

\begin{tabular}{cc}
$\mathrm{K}_{3} \mathrm{PO}_{4}$ & $\mathrm{IL}$ \\
molality & molality \\
\hline 0.5823 & 5.3224 \\
0.7506 & 3.4468 \\
0.8316 & 3.0543 \\
0.8789 & 2.8677 \\
1.0155 & 2.5716 \\
1.1041 & 2.3434 \\
1.2143 & 2.1437 \\
1.3899 & 1.8879 \\
1.6828 & 1.5161 \\
2.1400 & 1.0191 \\
\hline
\end{tabular}


Table S2 Phase diagram data of $[\mathrm{Ch}]\left[\mathrm{C}_{5} \mathrm{H}_{11} \mathrm{COO}\right]$

\begin{tabular}{cc}
$\mathrm{K}_{3} \mathrm{PO}_{4}$ & $\mathrm{IL}$ \\
molality & molality \\
\hline 0.4797 & 4.2315 \\
0.6349 & 3.5163 \\
0.9516 & 2.7278 \\
1.0346 & 2.4655 \\
1.2025 & 2.1557 \\
1.3413 & 1.9103 \\
1.4632 & 1.7131 \\
1.5942 & 1.5249 \\
1.6936 & 1.3683 \\
1.8219 & 1.1919 \\
1.9363 & 1.0407 \\
2.0650 & 0.9035 \\
2.1434 & 0.8109 \\
2.2409 & 0.7114 \\
\hline
\end{tabular}


Table S3 Phase diagram data of $[\mathrm{Ch}]\left[\mathrm{C}_{7} \mathrm{H}_{15} \mathrm{COO}\right]$

\begin{tabular}{|c|c|c|c|}
\hline $\begin{array}{c}\mathrm{K}_{3} \mathrm{PO}_{4} \\
\text { molality }\end{array}$ & $\begin{array}{c}\text { IL } \\
\text { molality }\end{array}$ & $\begin{array}{c}\mathrm{K}_{3} \mathrm{PO}_{4} \\
\text { molality }\end{array}$ & $\begin{array}{c}\text { IL } \\
\text { molality }\end{array}$ \\
\hline 0.4052 & 5.9120 & 1.8925 & 0.8822 \\
\hline 0.5799 & 5.1471 & 1.9294 & 0.8289 \\
\hline 0.6349 & 4.4589 & 1.9628 & 0.7867 \\
\hline 0.7488 & 3.9955 & 1.9893 & 0.7510 \\
\hline 0.7539 & 3.5201 & 2.0145 & 0.7185 \\
\hline 0.8785 & 3.1805 & 2.0390 & 0.6840 \\
\hline 0.9651 & 2.9137 & 2.0724 & 0.6474 \\
\hline 1.0303 & 2.6929 & 2.0998 & 0.6147 \\
\hline 1.1035 & 2.4921 & 2.1241 & 0.5869 \\
\hline 1.1539 & 2.3299 & 2.1456 & 0.5584 \\
\hline 1.2261 & 2.1453 & 2.1717 & 0.5293 \\
\hline 1.3107 & 1.9683 & 2.1996 & 0.5017 \\
\hline 1.3607 & 1.8306 & 2.2283 & 0.4804 \\
\hline 1.4271 & 1.6829 & 2.2341 & 0.4659 \\
\hline 1.5274 & 1.5613 & 2.2574 & 0.4447 \\
\hline 1.5262 & 1.5048 & 2.2825 & 0.4236 \\
\hline 1.5848 & 1.3944 & 2.3065 & 0.4039 \\
\hline 1.6379 & 1.3019 & 2.3261 & 0.3884 \\
\hline 1.7010 & 1.2049 & 2.3344 & 0.3762 \\
\hline 1.7352 & 1.1368 & 2.3547 & 0.3587 \\
\hline 1.7792 & 1.0595 & 2.4189 & 0.3135 \\
\hline 1.8269 & 0.9915 & 2.5006 & 0.2612 \\
\hline 1.8560 & 0.9393 & 2.5291 & 0.2447 \\
\hline
\end{tabular}


Table S4 Phase diagram data of $[\mathrm{Ch}]\left[\mathrm{C}_{11} \mathrm{H}_{23} \mathrm{COO}\right]$

\begin{tabular}{cccc}
\hline $\mathrm{K}_{3} \mathrm{PO}_{4}$ & $\begin{array}{c}\mathrm{IL} \\
\text { molality }\end{array}$ & $\begin{array}{c}\mathrm{K}_{3} \mathrm{PO}_{4} \\
\text { molality }\end{array}$ & $\begin{array}{c}\mathrm{IL} \\
\text { molality }\end{array}$ \\
\hline 1.7481 & 0.9301 & 2.0374 & 0.4651 \\
1.7572 & 0.8529 & 2.0468 & 0.4520 \\
1.8097 & 0.8165 & 2.0655 & 0.4407 \\
1.8125 & 0.7723 & 2.0681 & 0.4295 \\
1.8483 & 0.7415 & 2.0760 & 0.4197 \\
1.8586 & 0.7165 & 2.0843 & 0.4087 \\
1.8791 & 0.6976 & 2.0998 & 0.3966 \\
1.8978 & 0.6664 & 2.1221 & 0.3826 \\
1.9182 & 0.6417 & 2.1410 & 0.3581 \\
1.9024 & 0.6257 & 2.1511 & 0.3514 \\
1.9425 & 0.6054 & 2.1659 & 0.3348 \\
1.9470 & 0.5701 & 2.1695 & 0.3206 \\
1.9837 & 0.5445 & 2.1892 & 0.2954 \\
1.9933 & 0.5160 & 2.1929 & 0.2861 \\
2.0038 & 0.4988 & 2.2122 & 0.2784 \\
2.0110 & 0.4830 & & \\
\hline
\end{tabular}


Table S5 Phase Diagram Fitting Parameters

\begin{tabular}{ccccc}
\hline & $A$ & $B$ & $C$ & $R^{2}$ \\
\hline$[\mathrm{Ch}]\left[\mathrm{C}_{3} \mathrm{H}_{7} \mathrm{COO}\right]$ & $127.5 \pm 7.2$ & $-0.4168 \pm 0.0195$ & $6.631 \times 10-6 \pm 3.947 \times 10-6$ & 0.9965 \\
{$[\mathrm{Ch}]\left[\mathrm{C}_{5} \mathrm{H}_{11} \mathrm{COO}\right]$} & $82.04 \pm 0.72$ & $-0.2601 \pm 0.0030$ & $3.000 \times 10^{-5} \pm 0.054 \times 10^{-5}$ & 0.9999 \\
{$[\mathrm{Ch}]\left[\mathrm{C}_{7} \mathrm{H}_{15} \mathrm{COO}\right]$} & $96.90 \pm 1.25$ & $-0.2753 \pm 0.0046$ & $4.000 \times 10^{-5} \pm 0.078 \times 10^{-5}$ & 0.9992 \\
{$[\mathrm{Ch}]\left[\mathrm{C}_{11} \mathrm{H}_{23} \mathrm{COO}\right]$} & $14.81 \pm 6.86$ & $0.2130 \pm 0.1094$ & $8.000 \times 10^{-5} \pm 0.538 \times 10^{-5}$ & 0.9984 \\
\hline
\end{tabular}

Table S6 Compositions of IL-/salt- rich phases of ABS (mass fraction, \%)

\begin{tabular}{cccccc}
\hline & {$[\mathrm{IL}]_{\mathrm{IL}}$} & {$[\text { salt }]_{\mathrm{IL}}$} & {$[\mathrm{IL}]_{\text {salt }}$} & {$[\text { salt }]_{\text {salt }}$} & TLL \\
\hline$[\mathrm{Ch}]\left[\mathrm{C}_{3} \mathrm{H}_{7} \mathrm{COO}\right]$ & 30.92 & 11.40 & 7.43 & 36.54 & 34.40 \\
{$[\mathrm{Ch}]\left[\mathrm{C}_{5} \mathrm{H}_{11} \mathrm{COO}\right]$} & 45.72 & 4.99 & 3.33 & 37.69 & 53.53 \\
{$[\mathrm{Ch}]\left[\mathrm{C}_{7} \mathrm{H}_{15} \mathrm{COO}\right]$} & 49.71 & 5.75 & 1.25 & 40.24 & 59.48 \\
{$[\mathrm{Ch}]\left[\mathrm{C}_{7} \mathrm{H}_{15} \mathrm{COO}\right]^{*}$} & 37.75 & 10.59 & 1.99 & 37.96 & 45.03 \\
{$[\mathrm{Ch}]\left[\mathrm{C}_{11} \mathrm{H}_{23} \mathrm{COO}\right]^{* \mathrm{a}}$} & - & - & - & - & - \\
\hline
\end{tabular}

${ }^{a}$ Reliable calculation results cannot be obtained because of the narrow concentration range in the phase diagram. 
Part C: Solvatochromic Parameters

Table S7 $\pi^{*}$ and $\beta$ values of IL-rich phases

\begin{tabular}{cccccc}
\hline & {$[\mathrm{Ch}]\left[\mathrm{C}_{3} \mathrm{H}_{7} \mathrm{COO}\right]$} & {$[\mathrm{Ch}]\left[\mathrm{C}_{5} \mathrm{H}_{11} \mathrm{COO}\right]$} & {$\left[\mathrm{C}_{7} \mathrm{H}_{15} \mathrm{COO}\right]$} & {$\left[\mathrm{C}_{7} \mathrm{H}_{15} \mathrm{COO}\right]^{*}$} & {$\left[\mathrm{C}_{11} \mathrm{H}_{23} \mathrm{COO}\right]^{*}$} \\
\hline$\pi^{*}$ & 1.303 & 1.197 & 0.938 & 0.944 & 0.843 \\
$\beta$ & 0.393 & 0.557 & 0.884 & 0.798 & 0.752 \\
\hline
\end{tabular}




\section{Part D: Molar Absorptivity}

Table S8 Molar absorptivity of solutes in different phases

\begin{tabular}{|c|c|c|c|c|c|}
\hline \multirow{4}{*}{$\begin{array}{l}\text { Hydrophobic IL- } \\
\text { water systems }\end{array}$} & & & Phe & Trp & Caf \\
\hline & \multirow{2}{*}{$\begin{array}{c}\text { IL } \\
\text { phase }\end{array}$} & {$[\mathrm{BMIm}]\left[\mathrm{PF}_{6}\right]$} & 158 & $4960 \pm 36$ & $7873 \pm 76$ \\
\hline & & {$[\mathrm{BMIm}]\left[\mathrm{Tf}_{2} \mathrm{~N}\right]$} & 155 & $4900 \pm 45$ & $7533 \pm 73$ \\
\hline & \multicolumn{2}{|c|}{ Water phase } & $146 \pm 1$ & $4811 \pm 38$ & $7377 \pm 112$ \\
\hline \multirow{9}{*}{$\begin{array}{c}\text { Aqueous biphasic } \\
\text { systems }\end{array}$} & \multirow{8}{*}{$\begin{array}{l}\text { IL-rich } \\
\text { phase }\end{array}$} & {$[\mathrm{BMIm}] \mathrm{Br}$} & $227 \pm 1$ & $10517 \pm 21$ & $10211 \pm 24$ \\
\hline & & {$[\mathrm{BMIm}] \mathrm{Cl}$} & $227 \pm 1$ & $10543 \pm 20$ & $10208 \pm 21$ \\
\hline & & {$[\mathrm{EMIm}] \mathrm{CF}_{3} \mathrm{SO}_{3}$} & 227 & $10491 \pm 78$ & $10179 \pm 17$ \\
\hline & & {$[\mathrm{Ch}]\left[\mathrm{C}_{3} \mathrm{H}_{7} \mathrm{COO}\right]$} & 226 & $10370 \pm 19$ & $10087 \pm 37$ \\
\hline & & {$[\mathrm{Ch}]\left[\mathrm{C}_{5} \mathrm{H}_{11} \mathrm{COO}\right]$} & $224 \pm 1$ & $10335 \pm 23$ & $10001 \pm 54$ \\
\hline & & {$[\mathrm{Ch}]\left[\mathrm{C}_{7} \mathrm{H}_{15} \mathrm{COO}\right]$} & $224 \pm 1$ & $10233 \pm 24$ & $9912 \pm 25$ \\
\hline & & {$[\mathrm{Ch}]\left[\mathrm{C}_{11} \mathrm{H}_{23} \mathrm{COO}\right]$} & $229 \pm 7$ & $10347 \pm 47$ & $10321 \pm 110$ \\
\hline & & {$\left[\mathrm{P}_{4444}\right]\left[\mathrm{C}_{11} \mathrm{H}_{23} \mathrm{COO}\right]$} & 226 & $10303 \pm 18$ & $10393 \pm 71$ \\
\hline & \multicolumn{2}{|c|}{ Salt-rich phase } & $226 \pm 4$ & $10231 \pm 12$ & $9805 \pm 100$ \\
\hline
\end{tabular}

IL phases and water phases of hydrophobic IL-water systems were diluted with methanol and ILrich phases and salt-rich phases were diluted with purified water. All the molar absorptivity values were presented in $\mathrm{kg} \cdot \mathrm{mol}^{-1} \cdot \mathrm{cm}^{-1}$ because concentrations of the samples were calculated with mass fraction. 